\title{
Deformation and vibration of an oblique elliptic torus
}

\author{
Bo-Hua Sun ${ }^{1}$ \\ ${ }^{1}$ Institute of Mechanics and Technology 8 School of Civil Engineering, \\ Xi'an University of Architecture and Technology, Xi'an 710055, China \\ http://imt.xauat.edu.cn \\ email: sunbohua@xauat.edu.cn
}

\begin{abstract}
The formulation used by the most of studies on an elastic torus are either Reissner mixed formulation or Novozhilov's complex-form one, however, for vibration and some displacement boundary related problem of the torus, application of those formulations has encountered great difficulty. It is highly demanded to have a displacement-type formulation for the torus. In this paper, I will simulate some typical problems and free vibration of the torus. The numerical results are verified by both finite element analysis and H. Reissner's formulation. My investigations show that both deformation and stress response of an elastic torus are sensitive to the radius ratio, and suggest that the analysis of a torus should be done by using the bending theory of a shell, and also reveal that the inner torus is stronger than outer torus due to the property of their Gaussian curvature. Regarding the free vibration of the torus, our analysis indicates that both initial in $u$ and $w$ direction must be included otherwise will cause big errors in eigenfrequency.
\end{abstract}

Keywords: elliptic torus, oblique, deformation, vibration, Gauss curvature, Maple

\section{INTRODUCTION}

Many natural and man-made objects take the shape of torus, doughnut and tube might be two of most popular torus ( shown in Fig. 1). Among regular shells, such as circular cylindrical shells, conical shells, spherical shells, and tori, the deformation of the torus is one of the most difficulty topics due to its complicated topology, where the torus geometry has both Positive, negative Gaussian curvature and turning points.

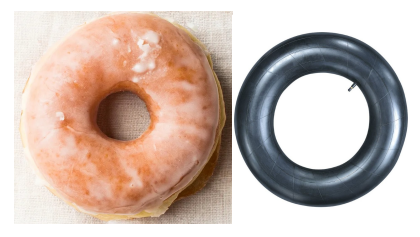

FIG. 1: Doughnut and tube.

The torus has been studied for more than 110 years (Chang (Zhang) 1949 [1], Qian and Liang 1979 [2], Xia and Zhang 1986 [3], Zhang, Ren and Sun 1990 [4], Zhang and Zhang 1991 [5], 1994 [6], Audoly and Pomeau 2002 [7], 2010 [8], Sun 2010 [9] 2012 [10], Clark 1950 [11, 12], Dahl 1953 [13], Novozhilov 1959 [14], Timoshenko and Woinowsky-Krieger 1959 [15], Flügge 1973 [16], Gol'denveizer 1961 [17] Sun 2013 [18], Föppl 1907 [19] Weihs 1911 [20], Wissler 1916 [21], Kuznetsov and Levyakov 2001 [22], Zingoni, Enoma and Govender 2015 [23], Jiammeepreecha and Chucheepsakul 2017 [24], Enoma and Zingoni 2020 [25], H. Reissner 1912 [26], Meissner 1915 [27], Tölke 1938 [28], E. Reissner 1949 [29], Tao 1959 [30], Steele 1965 [31], Sun 2018 [32], Sun 2020 [33]). The most of publications have been focused on circular torus, and only small percentage on non-circular cross-section torus [22-25], in which, Zingoni, Enoma and Govender
2015 [23] considered a semi-elliptic toroid and tried to get an approximate bending solution that may be valid in regions adjacent to the horizontal equatorial plane.

When the torus was first studied, high-order and complicated governing equations of a torus under symmetric loads were reduced to lower-order, ordinary differential equation (ODE) by Hans Reissner (1912)[26] when he was a professor at ETH in Switzerland. His colleague at ETH, Meissner (1915) [27] derived a complex-form equations for the shell of revolution. Hence, the first complex-form equation of the shells of revolution including torus is called the Reissner-Meissner equation, which is a ODE system about shear force $Q$ and rotation $\chi$ (Flügge 1973 [16]). In 1959, Novozhilov published his celebrated monograph on the complex-form theory of shells, and formulated symmetrical deformation of a torus in his complex-form (Novozhilov 1959 [14]).

Regarding formulation of elastic torus, governing equations that all publications adopted are either ReissnerMeissner's mixed formulation or Novozhilov's complexform one. Although those formulations have some advantages, however they can not be used for vibration analysis and/or have difficulty when you have to deal with displacement boundary conditions. Therefore, it is desired to have displacement formulations for elastic torus. Sun 2010 [9] who derived displacement-type equations for torus and obtained a closed-form solution for special case as radius ratio $\frac{a}{R} \rightarrow 0$. This special solution can not capture full mechanics nature of torus and therefore are needed to be improved further.

In corresponding to elliptic torus, to my best of knowledge, no any research on the oblique elliptic torus has been seen in the literature. In this paper, we will apply the displacement formulation to solve the strength and vibration of oblique elliptic torus.

This study wishes to carry on my previous works (Sun 2010 [9]), and investigates mechanical response for ar- 
bitrary ratio $\frac{a}{R}$. The content is organised as follows: Section 2 derives displacement-type formulation of torus. Section 3 applies the displacement formulation to oblique elliptic torus. Section 4 applies the displacement formulation to normal elliptic torus. Section 5 verifies our results by both finite element analysis and Hans Reissner's formulation. Section 6 provides conclusions.

\section{DISPLACEMENT-TYPE FORMULATION OF SYMMETRICAL DEFORMATION OF ELASTIC TORUS}

For convenience of readers, all quantities notation and definition please refers to well-known book of J.N. Reddy 2007 [37]. For the torus shown in Fig. 2, the positions of points on the middle surface will be determined by the angles $\theta$ and $\varphi$. Further, let $r_{1}$ be the radius of curvature of the meridian and $r_{2}$ the radius of curvature of the normal section, tangential to the parallel circle. This second radius is equal to the segment of the perpendicular to the middle surface between this surface and the axis of the torus.

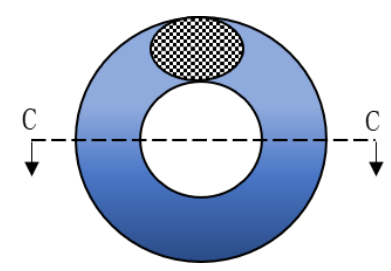

(a)

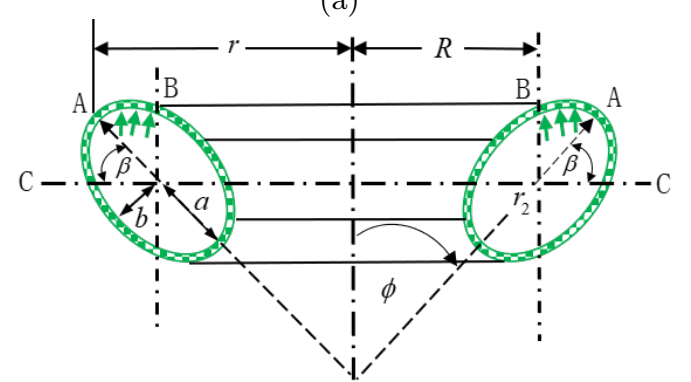

(b)

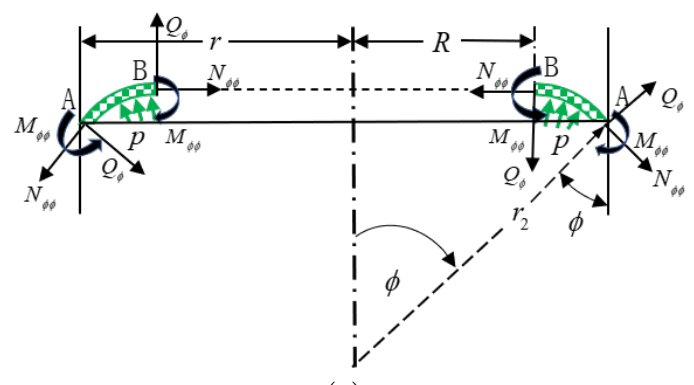

(c)

FIG. 2: Geometry of torus, loading, forces and moments.

The principal radii of curvature of the oblique elliptic torus in Fig.2 are given by

$$
\begin{aligned}
& r_{1}=\frac{1}{\cos \beta} \frac{a^{2} b^{2}}{\left(a^{2} \sin ^{2} \phi+b^{2} \cos ^{2} \phi\right)^{3 / 2}} \\
& r_{2}=\frac{R}{\sin \phi}+\frac{1}{\cos \beta} \frac{a^{2}}{\left(a^{2} \sin ^{2} \phi+b^{2} \cos ^{2} \phi\right)^{1 / 2}},
\end{aligned}
$$

where $\beta$ is oblique angle. From the above relations, we can see that the oblique angle will strongly influence on the mechanics of elliptic torus via affecting the main principal radii $r_{2}$. The above radii will be reduced to normal elliptic torus when $\beta=0$.

Regarding the forces shown in Fig.2, the balance equations are

$$
\begin{aligned}
\frac{d}{d \phi}\left(r N_{\phi \phi}\right)-N_{\theta \theta} r_{1} \cos \phi+r Q_{\phi}+r_{1} r f_{\phi} & =0 \\
\frac{d}{d \phi}\left(r Q_{\phi}\right)-r_{1} r\left(\frac{N_{\phi \phi}}{r_{1}}+\frac{N_{\theta \theta}}{r_{2}}\right)+r_{1} r f_{\zeta} & =0
\end{aligned}
$$

where $r=r_{2} \sin \phi$, distributed loads $f_{\phi}, f_{\zeta}$ along $\phi, \zeta$ direction, and shear force

$$
Q_{\phi}=\frac{1}{r_{1} r} \frac{d}{d \phi}\left(r M_{\phi \phi}\right)-\frac{1}{r} \cos \phi M_{\theta \theta} .
$$

where the resultant membranae forces are $N_{\phi \phi}=$ $K\left(\varepsilon_{\phi \phi}+\nu \varepsilon_{\theta \theta}\right), N_{\theta \theta}=K\left(\nu \varepsilon_{\phi \phi}+\varepsilon_{\theta \theta}\right)$, and resultan$\mathrm{t}$ bending moments are $M_{\phi \phi}=B\left(\kappa_{\phi \phi}+\nu \kappa_{\theta \theta}\right), M_{\theta \theta}=$ $B\left(\nu \kappa_{\phi \phi}+\kappa_{\theta \theta}\right)$; The membranae strains are $\varepsilon_{\phi \phi}=\frac{1}{r_{1}}\left(\frac{d u}{d \phi}+\right.$ $w), \varepsilon_{\theta \theta}=\frac{1}{r_{2}}(u \cot \phi+w)$, and change of curvature are $\kappa_{\phi \phi}=\frac{1}{r_{1}} \frac{d \varphi_{\phi}}{d \phi}, \kappa_{\theta \theta}=\frac{\cot \phi}{r_{2}} \varphi_{\phi}$, where total rotation $\varphi_{\phi}=\frac{1}{r_{1}}\left(u-\frac{d w}{d \phi}\right)$; and membranae stiffness $K=\frac{E h}{1-\nu^{2}}$, bending stiffness $B=\frac{E h^{3}}{12\left(1-\nu^{2}\right)}$, thickness $h$, Young modulus $E$ and Poisson's ratio $\nu$.

With the above strains and curvature change, the resultant membranae force and bending moments can be expressed in terms of displacements $u, w$ as follows;

$$
\begin{aligned}
N_{\phi \phi} & =K\left[\frac{1}{r_{1}}\left(\frac{d u}{d \phi}+w\right)+\nu \frac{1}{r_{2}}(u \cot \phi+w)\right], \\
N_{\theta \theta} & =K\left[\nu \frac{1}{r_{2}}(u \cot \phi+w)+\frac{1}{r_{1}}\left(\frac{d u}{d \phi}+w\right)\right], \\
M_{\phi \phi} & =B\left[\frac{1}{r_{1}} \frac{d \varphi_{\phi}}{d \phi}+\nu \frac{\cot \phi}{r_{2}} \varphi_{\phi}\right] \\
& =B\left[\frac{1}{r_{1}} \frac{d}{d \phi}\left[\frac{1}{r_{1}}\left(u-\frac{d w}{d \phi}\right)\right]+\nu \frac{\cot \phi}{r_{2}} \frac{1}{r_{1}}\left(u-\frac{d w}{d \phi}\right)\right] \\
M_{\theta \theta} & =B\left[\nu \frac{1}{r_{1}} \frac{d \varphi_{\phi}}{d \phi}+\frac{\cot \phi}{r_{2}} \varphi_{\phi}\right] \\
& =B\left[\nu \frac{1}{r_{1}} \frac{d}{d \phi}\left[\frac{1}{r_{1}}\left(u-\frac{d w}{d \phi}\right)\right]+\frac{\cot \phi}{r_{2}} \frac{1}{r_{1}}\left(u-\frac{d w}{d \phi}\right)\right]
\end{aligned}
$$

Substituting the shear force $Q_{\phi}$ in Eq.3 into Eq.2 and 
produce

$$
\begin{gathered}
\frac{d}{d \phi}\left(r N_{\phi \phi}\right)-N_{\theta \theta} r_{1} \cos \phi \\
+\frac{1}{r_{1}}\left[\frac{d}{d \phi}\left(r M_{\phi \phi}\right)-r \cos \phi M_{\theta \theta}\right]+r_{1} r f_{\phi}=0 \\
\frac{d}{d \phi}\left[\frac{1}{r} \frac{d}{d \phi}\left(r M_{\phi \phi}\right)-\cos \phi M_{\theta \theta}\right] \\
-r_{1} r\left(\frac{N_{\phi \phi}}{r_{1}}+\frac{N_{\theta \theta}}{r_{2}}\right)+r_{1} r f_{\zeta}=0
\end{gathered}
$$

The Eq. 5 can be further simplified by substituting the constitutive relations into Eq.bal-2, which will generate a final equations that is a six order ordinary differential equation system about displacement $u(\phi)$ and $w(\phi)$.

$$
\begin{gathered}
K \frac{d}{d \phi}\left[r\left[\frac{1}{r_{1}}\left(\frac{d u}{d \phi}+w\right)+\nu \frac{1}{r_{2}}(u \cot \phi+w)\right]\right] \\
-K r_{1} \cos \phi\left[\nu \frac{1}{r_{2}}(u \cot \phi+w)+\frac{1}{r_{1}}\left(\frac{d u}{d \phi}+w\right)\right] \\
+\frac{B}{r_{1}} \frac{d}{d \phi}\left(r\left[\frac{1}{r_{1}} \frac{d}{d \phi}\left[\frac{1}{r_{1}}\left(u-\frac{d w}{d \phi}\right)\right]+\nu \frac{\cot \phi}{r_{2}} \frac{1}{r_{1}}\left(u-\frac{d w}{d \phi}\right)\right]\right) \\
-\frac{B}{r_{1}} r \cos \phi\left[\nu \frac{1}{r_{1}} \frac{d}{d \phi}\left[\frac{1}{r_{1}}\left(u-\frac{d w}{d \phi}\right)\right]+\frac{\cot \phi}{r_{2}} \frac{1}{r_{1}}\left(u-\frac{d w}{d \phi}\right)\right] \\
B \frac{d}{d \phi}\left[\frac{1}{r} \frac{d}{d \phi}\left(r\left[\frac{1}{r_{1}} \frac{d}{d \phi}\left[\frac{1}{r_{1}}\left(u-\frac{d w}{d \phi}\right)\right]+\nu \frac{\cot \phi}{r_{2}} \frac{1}{r_{1}}\left(u-\frac{d w}{d \phi}\right)\right]\right)\right. \\
\left.-\cos \phi\left[\nu \frac{1}{r_{1}} \frac{d}{d \phi}\left[\frac{1}{r_{1}}\left(u-\frac{d w}{d \phi}\right)\right]+\frac{\cot \phi}{r_{2}} \frac{1}{r_{1}}\left(u-\frac{d w}{d \phi}\right)\right]\right] \\
-r K\left[\frac{1}{r_{1}}\left(\frac{d u}{d \phi}+w\right)+\nu \frac{1}{r_{2}}(u \cot \phi+w)\right] \\
-K r \frac{r_{1}}{r_{2}}\left[\nu \frac{1}{r_{2}}(u \cot \phi+w)+\frac{1}{r_{1}}\left(\frac{d u}{d \phi}+w\right)\right] \\
+r_{1} r f_{\zeta}=0,
\end{gathered}
$$

The above ODE system will be more complicated if taking into account of the principal radii. Obviously the ODE system has no analytical solution but numerical ones.

For simplification of presentation of our results, physical units will not be plotted in all drawings, all physical units are listed in Table I:

TABLE I: Physical units

\begin{tabular}{c|c|c|c|c|c|c|c|c|c|c|c}
\hline$R$ & $a, b$ & $R, h$ & $E$ & $\nu$ & $M_{\phi}$ & $N_{\phi}$ & $Q_{\phi}$ & $\sigma_{\phi}$ & $u$ & $w$ & $\Delta_{z}$ \\
\hline \hline$m$ & $m$ & $m$ & $N / m^{2}$ & 1 & $N m$ & $N / m$ & $N / m$ & $N / m^{2}$ & $m$ & $m$ & $m$ \\
\hline
\end{tabular}

\section{MECHANICS OF AN OBLIQUE ELLIPTIC TORUS}

In this section, we will apply the displacement formulation to the deformation analysis.

\section{A. Oblique elliptic torus with a penetrate cut}

Unless otherwise stated, numerical calculations in this paper are based on the data in Table I. We vary the oblique angle $\beta$, while other quantities are kept unchange.

For a complete torus with a penetrate cut along the parallel $\theta=\frac{\pi}{2}$ and loaded with vertical force $F$, the loading condition is shown in Fig. 11.

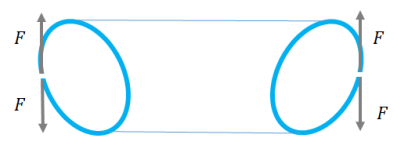

FIG. 3: Torus with a cut along its parallel at $\phi=\frac{\pi}{2}$ or $\phi=-\frac{\pi}{2}$ under load $F$

The boundary condition is:

$$
\begin{gathered}
\phi=\frac{\pi}{2}: N_{\phi \phi}=-\frac{F \cos \beta}{2 \pi\left(R+\frac{a}{\cos \beta}\right)}, \\
Q_{\phi}=-\frac{F \sin \beta}{2 \pi\left(R+\frac{a}{\cos \beta}\right)}, M_{\phi \phi}=0, \\
\phi=-\frac{3 \pi}{2}: N_{\phi \phi}=-\frac{F \cos \beta}{2 \pi\left(R+\frac{a}{\cos \beta}\right)}, \\
Q_{\phi}=\frac{F \sin \beta}{2 \pi\left(R+\frac{a}{\cos \beta}\right)}, M_{\phi \phi}=0 .
\end{gathered}
$$

A general code for this case is written by Maple [38]. With the help of the Maple code, some numerical results have been obtained and are shown in Fig. 8 .

The results indicate that all quantities such as bending moments, surface forces, shear force, and displacement are strongly effected by oblique angle.

\section{B. Deformation and stress caused by internal} pressure

For this problem, so far there has no a complete solution since no formulation can provide displacement period condition. The trick of this problem is that the periodic conditions must be used, namely $u(0)=u(2 \pi), w(0)=$ $w(2 \pi), \frac{d w}{d \phi}(0)=\frac{d w}{d \phi}(2 \pi), M_{\phi \phi}(0)=M_{\phi \phi}(2 \pi), N_{\phi \phi}(0)=$ $N_{\phi \phi}(2 \pi), Q_{\phi}(0)=Q_{\phi}(2 \pi)$. The results are presented in Fig.5.

The results indicate that all quantities such as bending moments, surface forces, shear force, and displacement are vary with the change of oblique angle.

\section{Free vibration of an oblique elliptic torus}

Besides finite element method, both Reissner's mixed formulation and complex-form one can not be used to deal with vibration of torus, because the acceleration of motion must be taken into account in the formulation. In 


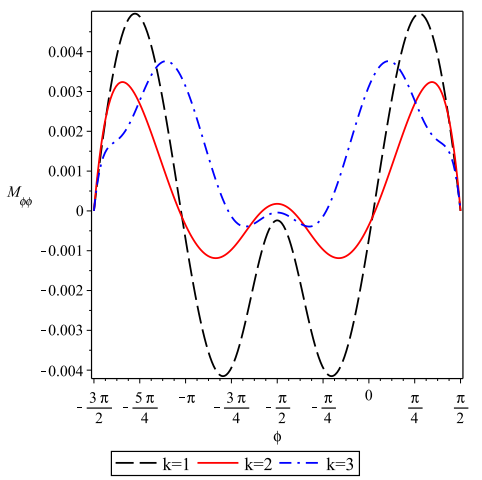

(a) Bending moment $M_{\phi \phi}$

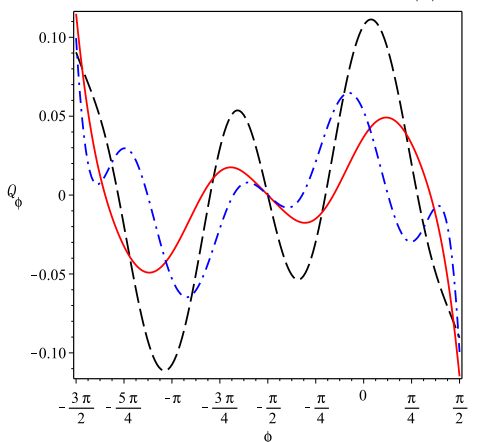

$--\mathrm{k}=1-\mathrm{k}=2-\cdot-\mathrm{k}=3$

(c) Shear force $Q_{\phi}$

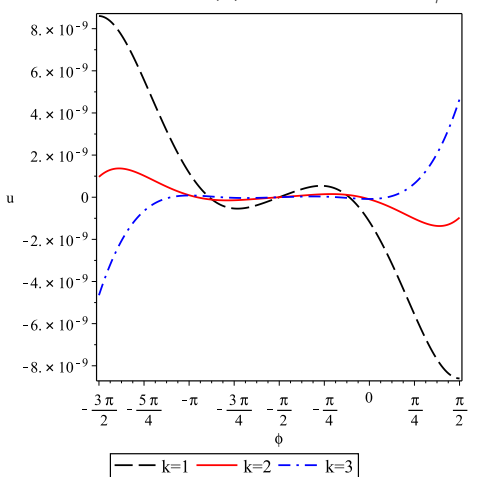

(e) $u$ Displacements in $\varphi$

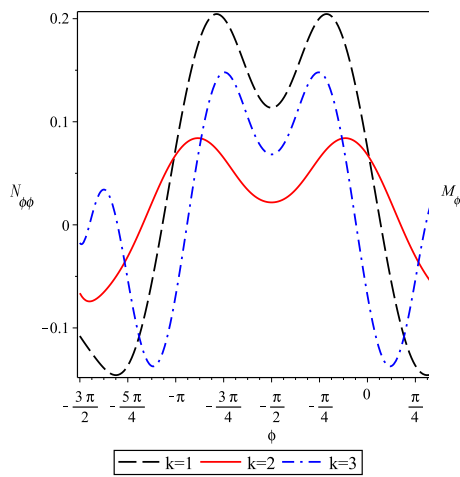

(b) Membranes force $N_{\phi \phi}$

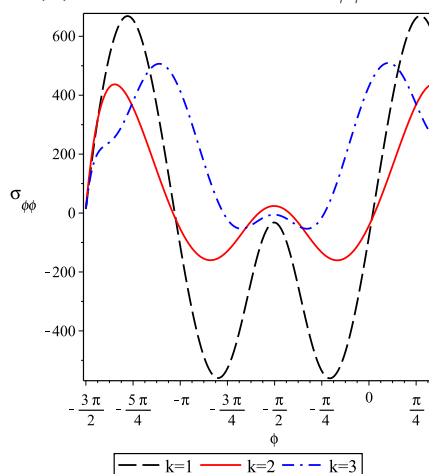

(d) Stress $\sigma_{\phi}$

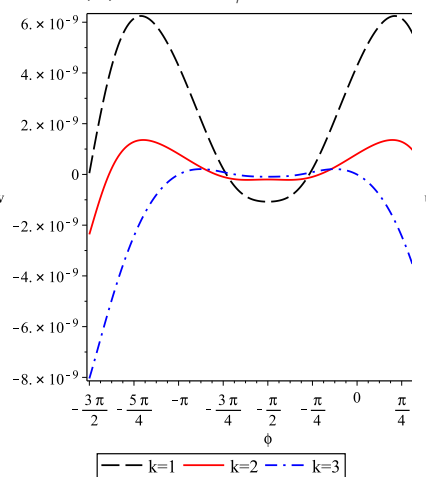

(f) Displacement $w$ in $\phi$ direction.

FIG. 4: Oblique elliptic torus with data: $a=0.1[m], b=$ $1.2 a[m], R=1[m], h=1 / 15[m], \beta=k \pi / 9, E=2.0 \times$ $10^{11} \mathrm{~N} / \mathrm{m}^{2}, \nu=0.3, F=1[N]$.

this case, displacement-type formulation is the only one can be accepted. Therefore, in this section, we will study symmetric free vibration of the torus. In this case, the initial namely $f_{\phi}=-\rho h \frac{\partial^{2} u}{\partial t^{2}}$ and $f_{\zeta}=-\rho h \frac{\partial^{2} w}{\partial t^{2}}$ must be introduced into Eq. 6 and lead to a free vibration equation system of oblique elliptic torus, where the mass density $\rho$ and time $t$.

For free vibration analysis, the displacement $u, w$ be set as $u=U(\phi) \exp (i \omega t)$ and $w=W(\phi) \exp (i \omega t)$, where $\omega$ is angular frequency, $U(\phi), W(\phi)$ are the eigenmode of $u, w$, respectively. We have simulated the torus as shown in Fig. 11 without load $F$, which means that we are dealing with free vibration. With our general Maple
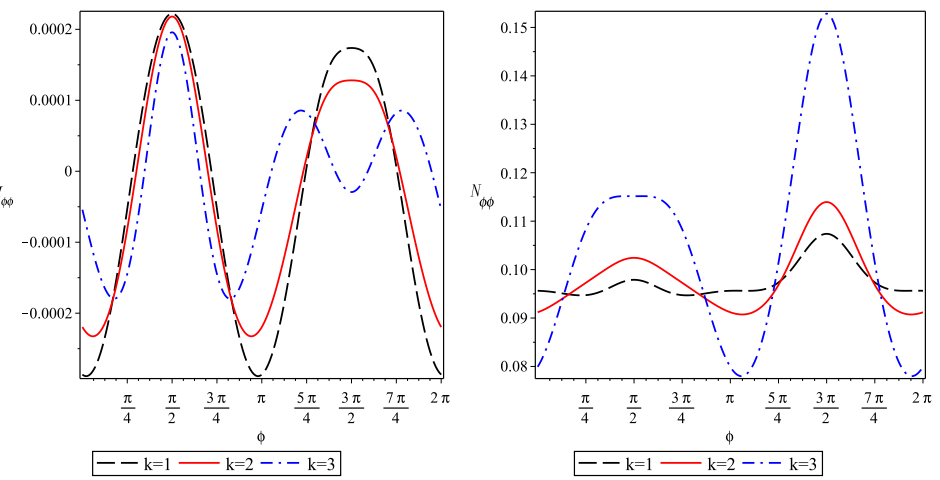

(a) Bending moment $M_{\phi \phi}$

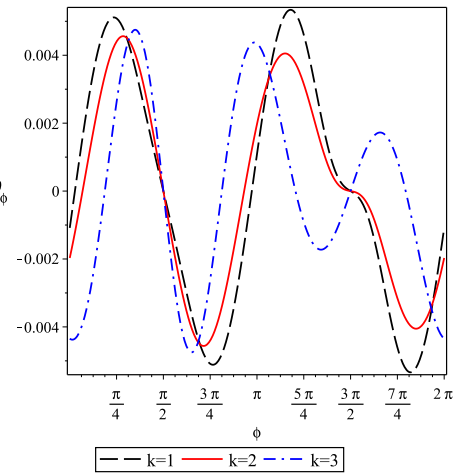

(b) Membranes force $N_{\phi \phi}$

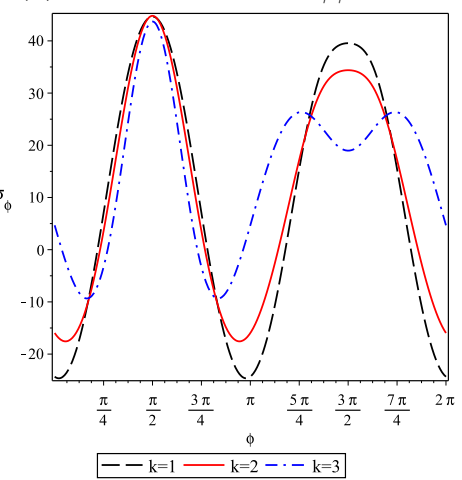

(c) Shear force $Q_{\phi}$

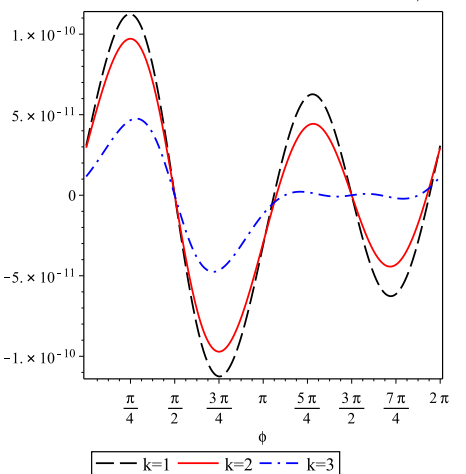

(d) Stress $\sigma_{\phi}$

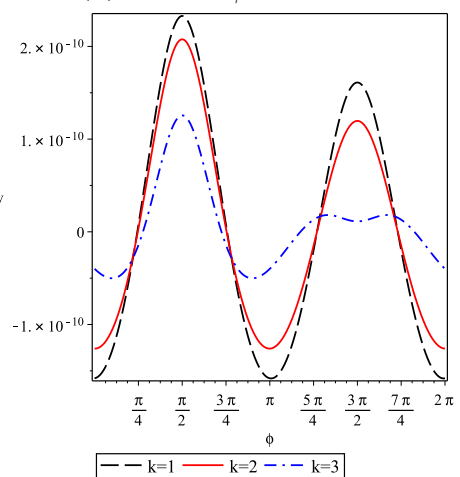

(e) $u$ Displacements in $\varphi$

(f) Displacement $w$ in $\phi$ direction.

FIG. 5: Deformation caused internal pressure. Oblique elliptic torus with data: $a=0.1[\mathrm{~m}], b=1.2 a[\mathrm{~m}], R=1[\mathrm{~m}], h=$ $1 / 15[m], \beta=k \pi / 9, E=2.0 \times 10^{11} \mathrm{~N} / \mathrm{m}^{2}, \nu=0.3, f_{\zeta}=$ $1\left[N / m^{2}\right]$.

code, eigenfrequencies are obtained.

(a)For free vibration of torus, if we take into account of acceleration in both direction of $u, w$, namely $f_{\phi}=$ $-\rho h \frac{\partial^{2} u}{\partial t^{2}}$ and $f_{\zeta}=-\rho h \frac{\partial^{2} w}{\partial t^{2}}$. The angular eigenfrequences are obtained as follows:

$$
\begin{gathered}
\beta=\pi / 6: \omega=367.843[1 / s] \\
\beta=2 \pi / 6: \omega=441.135[1 / s] .
\end{gathered}
$$

(b) For free vibration of torus, if we only take into account of acceleration in direction of $w$, namely $f_{\zeta}=$ 
$-\rho h \frac{\partial^{2} w}{\partial t^{2}}$. The eigenfrequency are obtained as follows:

$$
\begin{gathered}
\beta=\pi / 6: \omega=645.643[1 / s] \\
\beta=2 \pi / 6: \omega=660.330[1 / s] .
\end{gathered}
$$

The eigenmodes corresponding to the eigenfrequencies are

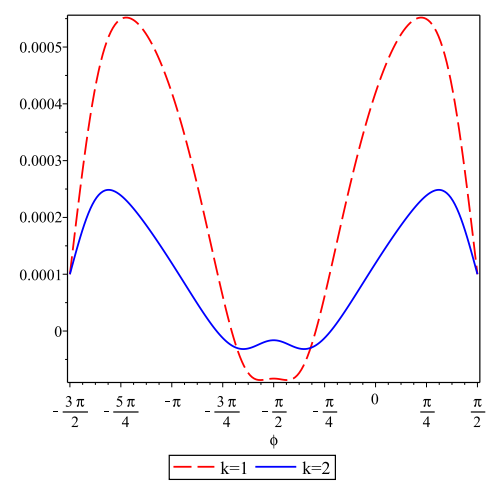

FIG. 6: Eigenmode of displacement $w$. The solid-line is for case (a) and point-line is for case (b).

The results indicate that the eigenfrequency in case of (a) and (b) has a big difference, while the eigenmode has little difference for literal motion $w$ and small off alignment for mid-surface motion $u$. The big frequency difference implies that the acceleration in u direction can not be omitted for vibration of torus.

It is worth to mention that such analysis has not been reported in the literature, other shell's vibration can be seen in a famous book by A W Leissa 1993. [39]

\section{MECHANICS OF A NORMAL ELLIPTIC TORUS}

If there were no oblique angle, namely $\beta=0$, all previous formulations will be reduced to normal elliptic torus as shown in Fig.7.

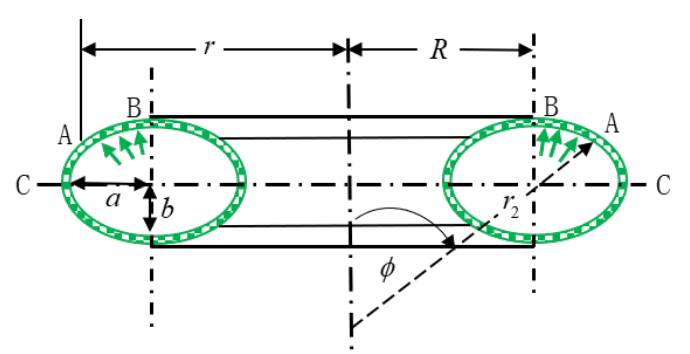

FIG. 7: Geometry of normal elliptic torus, loading, forces and moments.

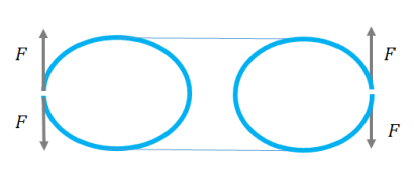

FIG. 8: Loaded with $F$.
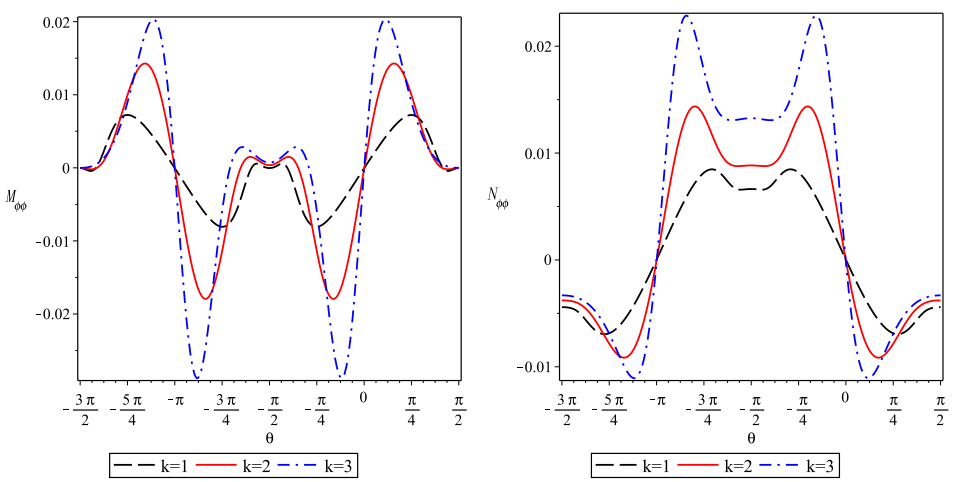

(a) Bending moment $M_{\phi \phi}$

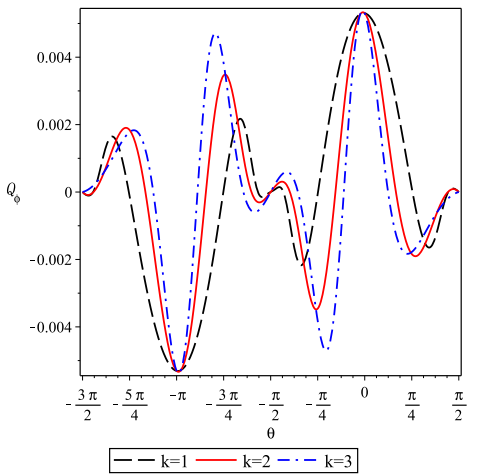

(b) Membranes force $N_{\phi \phi}$

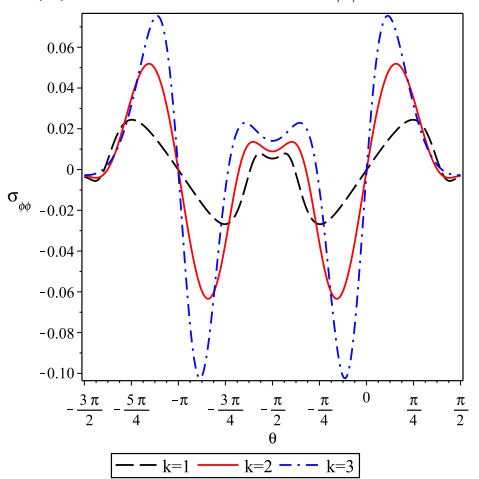

(c) Shear force $Q_{\phi}$

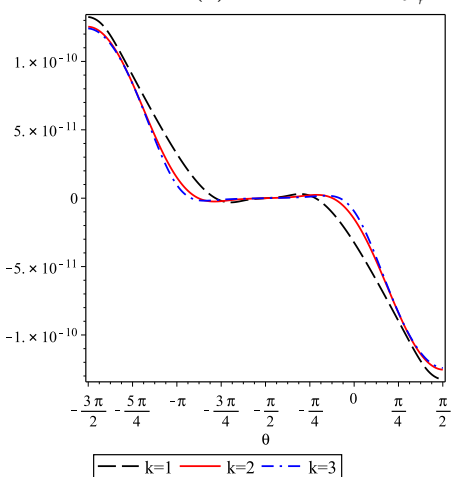

(d) Stress $\sigma_{\phi}$

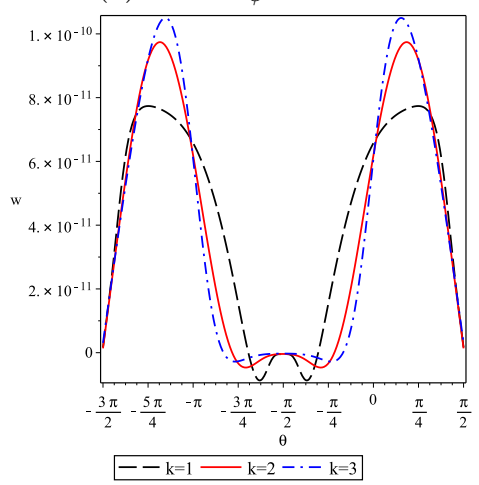

(e) $u$ Displacements in $\varphi$

(f) Displacement $w$ in $\phi$ direction.

FIG. 9: Deformation caused vertical force. Normal elliptic torus with data: $a=6 k[m], b=15, R=30[m], h=$ $a / 15[m], E=2.0 \times 10^{11} \mathrm{~N} / \mathrm{m}^{2}, \nu=0.3, F=1[N]$.

\section{A. Normal elliptic torus with a penetrate cut}

The above all figures indicate that all quantities such as bending moments, surface forces, shear force, and displacement are strongly effected by the radius ratio $\alpha=a / R$, and vary dramatically with $\phi$ both near to 
and far from the edge.

\section{B. Free vibration of a normal elliptic torus}

We have simulated the torus as shown in Fig. 8 without load $F$, which means that we are dealing with free vibration. For free vibration of torus, if we take into account of acceleration in both direction of $u, w$, namely $f_{\phi}=-\rho h \frac{\partial^{2} u}{\partial t^{2}}$ and $f_{\zeta}=-\rho h \frac{\partial^{2} w}{\partial t^{2}}$.

The eigenmodes corresponding and angular eigenfrequences are obtained and shown in Fig10.

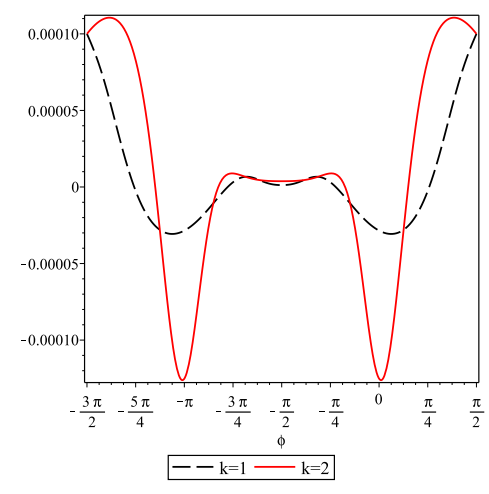

FIG. 10: Eigenmode of displacement $w$ : the dash-line is for $k=1$ and solid-line is for $k=2$; their corresponding angular eigenfrequences are $\omega=38.551[1 / \mathrm{s}]$ and $\omega=25.534$; Normal elliptic torus data: $a=10 k[m], \equiv 15[m], R=30[m], h=$ $a / 15[m], E=2.0 \times 10^{11} \mathrm{~N} / \mathrm{m}^{2}, \nu=0.3$.

\section{SIMULATION VALIDATION}

A. Displacement formulation vs. finite element analysis for circular torus

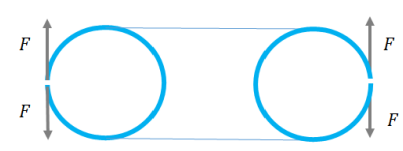

FIG. 11: Torus with a cut along its parallel at $\phi=\frac{\pi}{2}$ or $\phi=-\frac{\pi}{2}$ under load $F$

To verify our result, we carried out a finite element analysis for circular torus. The comparisons are shown in Fig. 12. The finite element results were simulated by ABAQUS with shell element S4R. We applied the boundary condition $\triangle_{z}(-3 \pi / 2)=0$ to cancel the rigid body motion, which might be the reason for minor differences in the range $\theta \in[-3 \pi / 2,-5 \pi / 4]$. From physics point of view, our result is symmetric respect to the axes of $\theta=-\pi / 2$ while the FEA has slightly symmetry breaking. Therefore, our results are more trustable since the
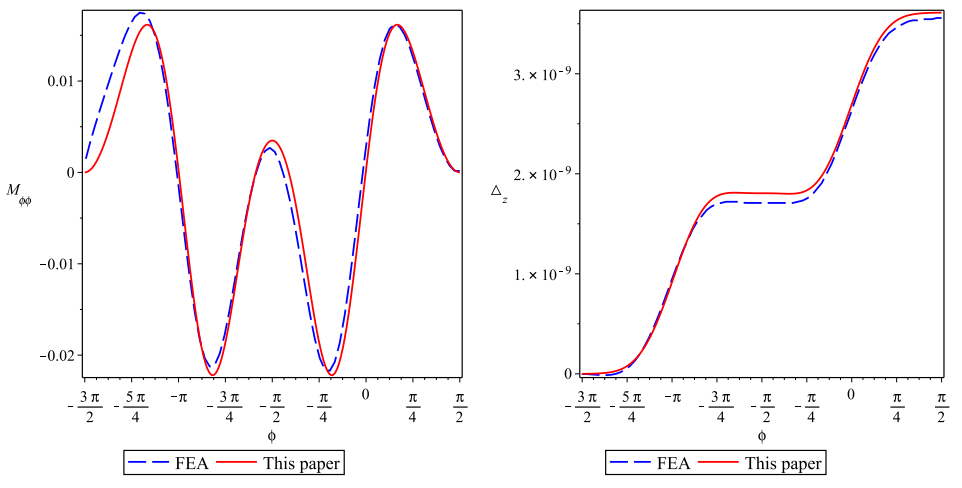

FIG. 12: Left: Bending moment $M_{\phi \phi}$; Right: Vertical displacement $\Delta_{z}$, this paper gives $\Delta_{z}(\pi / 2)=$ $3.61080495828841 \times 10^{-9}[\mathrm{~m}]$, FEM gives $\Delta_{z}(\pi / 2)=$ $3.557581142 \times 10^{-9}[\mathrm{~m}]$. Circular torus data: $a=$ $0.36[m], R=1[m], h=1 / 15[m], \beta=k \pi / 9, E=2.0 \times$ $10^{11} \mathrm{~N} / \mathrm{m}^{2}, \nu=0.3, F=1[N]$

finite element analysis supports our numerical prediction nicely.

\section{B. Comparison with Hans Reisnner's formulation on both normal and oblique elliptic torus}

In the theory of shells of revolution under axisymmetric load, according to Timoshenko and Woinowsky-Krieger 1959 [15], the decisive step was the introduction of $Q_{\phi}$ and $\chi$ as unknowns by H. Reissner 1912 [26]. The idea has been much extended by E. Meissner 1925 [27]. The formulation of Reissner-Meissner of shells of revaluation with constant thickness can be found in the masterpieces of Flügge 1973 [16]. All notations in this subsection can be found in Flügge's book [16].

The balance equations are:

$$
\begin{aligned}
& \frac{r_{2}}{r_{1}} \frac{d^{2} \chi}{d \phi^{2}}+\left[\frac{r_{2}}{r_{1}} \cot \phi+\frac{d}{d \phi}\left(\frac{r_{2}}{r_{1}}\right)\right] \frac{d \chi}{d \phi}-\left(\frac{r_{1}}{r_{2}} \cot ^{2} \phi+\mu\right) \chi \\
& =\frac{1}{B} r_{1} r_{2} Q_{\phi} \\
& \frac{r_{2}}{r_{1}} \frac{d^{2}}{d \phi^{2}}\left(r_{2} Q_{\phi}\right)+\left[\frac{r_{2}}{r_{1}} \cot \phi+\frac{d}{d \phi}\left(\frac{r_{2}}{r_{1}}\right)\right] \frac{d}{d \phi}\left(r_{2} Q_{\phi}\right) \\
& -\left(\frac{r_{1}}{r_{2}} \cot ^{2} \phi-\mu\right)\left(r_{2} Q_{\phi}\right) \\
& =-B\left(1-\mu^{2}\right) r_{1} \chi+P g(\phi)
\end{aligned}
$$

where $\chi$ is the angle by which an element $r d \phi$ of the meridian rotates during deformation; $r=r_{2} \sin \phi$, the load term $P$ is constant to be determined by the value of $N_{\phi \phi}(\pi / 2)=-\frac{P}{2 \pi r_{2}}$ and

$$
g(\phi)=\frac{1}{2 \pi \sin ^{2} \phi}\left[\frac{r_{1}^{2}-r_{2}^{2}}{r_{1} r_{2}} \cot \phi+\frac{d}{d \phi}\left(\frac{r_{2}}{r_{1}}\right)\right] .
$$

The resultant membranae forces $N_{\phi \phi}$ can be represented 
by shear force $Q_{\phi}$ :

$$
\begin{array}{r}
N_{\phi \phi}=-Q_{\phi} \cot \phi-\frac{P}{2 \pi r_{2}} \frac{1}{\sin ^{2} \phi}, \\
N_{\theta \theta}=-\frac{1}{r_{1}} \frac{d}{d \phi}\left(r_{2} Q_{\phi}\right)+\frac{P}{2 \pi r_{1}} \frac{1}{\sin ^{2} \phi} .
\end{array}
$$

Substituting the principal radii, ie., $r_{1}, r_{2}$ into Eq.13 will give governing equation for torus as follows:

$$
\begin{aligned}
& \frac{R+a \sin \phi}{a \sin \phi} \frac{d^{2} \chi}{d \phi^{2}}+\left[\frac{R+a \sin \phi}{a \sin \phi} \cot \phi+\frac{d}{d \phi}\left(\frac{R+a \sin \phi}{a \sin \phi}\right)\right] \frac{d \chi}{d \phi} \\
& -\left(\frac{a \sin \phi}{R+a \sin \phi} \cot ^{2} \phi+\mu\right) \chi=\frac{1}{B} a\left(a+\frac{R}{\sin \phi}\right) Q_{\phi}, \quad(17) \\
& \frac{R+a \sin \theta}{a \sin \phi} \frac{d^{2}}{d \phi^{2}}\left[\left(a+\frac{R}{\sin \phi}\right) Q_{\phi}\right] \\
& +\left[\frac{R+a \sin \phi}{a \sin \phi} \cot \phi+\frac{d}{d \phi}\left(\frac{R+a \sin \phi}{a \sin \phi}\right)\right] \frac{d}{d \phi}\left[\left(a+\frac{R}{\sin \phi}\right) Q_{\phi}\right] \\
& -\left(\frac{a \sin \phi}{R+a \sin \phi} \cot ^{2} \phi-\mu\right)\left(a+\frac{R}{\sin \phi}\right) Q_{\phi} \\
& =-B\left(1-\mu^{2}\right) a \chi+P g(\phi) .
\end{aligned}
$$

Once we obtain the shear force $Q_{\phi}$ and rotation $\chi$, one can compute all other quantities, such as $N_{\phi \phi}, M_{\phi \phi}$, as well as $u, w$. The problem of elastic torus becomes to find the shear force $Q_{\phi}$ and rotation $\chi$ from Eq.17 and 18. Obviously, Eq.17 and 18 are complicated and hard to be solved analytically.

For normal elliptic torus as shown in Fig.8, the comparison is given in Fig.13.

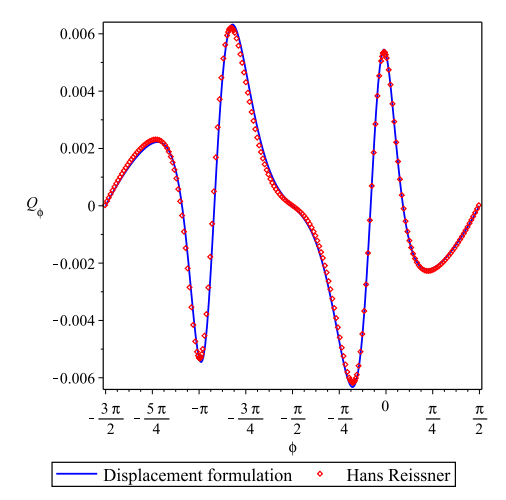

FIG. 13: Normal elliptic torus: displacement formulation vs. Hans Reissner. Problem as shown in Fig. 8. $a=$ $20[\mathrm{~m}], b=10[\mathrm{~m}], R=30[\mathrm{~m}], h=1 / 15[\mathrm{~m}], E=2.0 \times$ $10^{11} \mathrm{~N} / \mathrm{m}^{2}, \nu=0.3, F=1[N]$. Note: Hans Reissner formulation load $P$ and boundary condition: $P=F=1$ and $Q_{\phi}(-3 \pi / 2)=0, \frac{d \chi}{d \phi}(-3 \pi / 2)=0, Q_{\phi}(\pi / 2)=0, \frac{d \chi}{d \phi}(\pi / 2)=0$. Due to the difference of sign convention, $Q_{\phi}($ Displacementformulation $)=-Q_{\phi}($ Reissner-formulation $)$.

For oblique torus as shown in Fig.11, the comparison is given in Fig.14.

The numerical simulations show that displacement formulation agrees with Hans Reissner formulation very

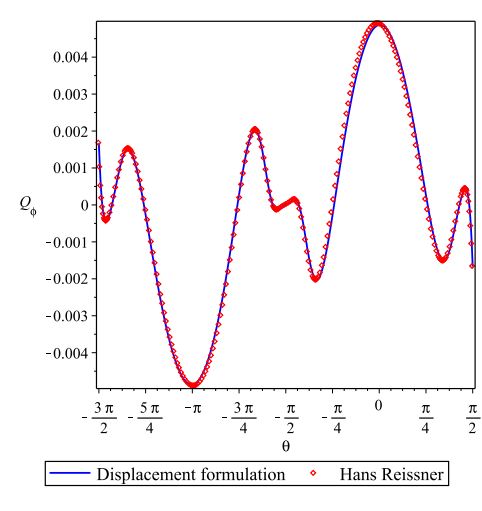

FIG. 14: Oblique elliptic torus: displacement formulation vs. Hans Reissner. Problem as shown in Fig. 11. $a=6[\mathrm{~m}], b=$ $15[m], R=30[m], h=6 / 15[m], E=2.0 \times 10^{11} \mathrm{~N} / \mathrm{m}^{2}, \nu=$ $0.3, F=1[N], \beta=\pi / 8$. Note: Hans Reissner formulation load $P$ and boundary condition: $P=F=1$ and $Q_{\phi}(-3 \pi / 2)=-\frac{F \sin \beta}{2 \pi\left(R+\frac{a}{\cos \beta}\right)}, \frac{d \chi}{d \phi}(-3 \pi / 2)=0, Q_{\phi}(\pi / 2)=$ $\frac{F \sin \beta}{2 \pi\left(R+\frac{a}{\cos \beta}\right)}, \frac{d \chi}{d \phi}(\pi / 2)=0$. Due to the difference of sign convention, $Q_{\phi}($ Displacement-formulation $)=-Q_{\phi}($ Reissnerformulation).

well, which provides a good supportive evidence about the correctness of our both formulation and simulation.

\section{CONCLUSIONS}

We have formulated elastic oblique elliptic torus in terms of displacement and successfully solved some typical problems, free vibration of the torus has been studied. To verify our formulation, we wrote a computational code in Maple and carried out some numerical simulations. The validation of our numerical results was confirmed and supported by both finite element analysis and Hans Reissner's formulation. Our investigations show that both deformation and stress response of an elastic torus are sensitive to the radius ratio and dramatically vary with the meridian angle, therefore the analysis of a torus should be done by the bending theory of a shell rather than membrane theory of shell. Our numerical studies also reveal that the inner torus is stronger than outer torus due to the property of their Gaussian curvature. Concerning the free vibration of torus, our analysis indicates that both initial in $\mathrm{u}$ and $\mathrm{w}$ direction must be included otherwise will cause big errors if initial in $u$ direction were omitted. Last but not least, the oblique angle has a strong influence on the mechanics of elliptic torus, because the oblique angle will affect the main principal radii.

\section{Acknowledgement:}

The author is honored to have benefits from my supervisor Prof. Dr.Ing Wei Zhang (Wei Chang ) at Tsinghua University, who was the first Chinese scholar studied the deformation of torus, it is my privilege to dedicate this paper to the memories of Prof. Zhang for 
his great contribution to the analysis of elastic torus. The author appreciates my students: Mr. Guang-Kai Song, for providing finite element analysis data in Fig. 12; Mr. Jie Wei, for preparation of Fig.2 (a,b).

Data availability The data that support the findings of this study are available from the corresponding author upon reasonable request.

Conflict of interest The author declares that he has no known competing financial interests or personal relationships that could have appeared to influence the work reported in this paper.
[1] W. Chang (W. Zhang), Derspannungszustand in kreisringschale und ähnlichenSchalen mit Scheitelkreisringen unter drehsymmetrischer Belastung. Arbeitzur Erlangung des Grades eines Doctor-Ingenieurs der Technichen Hochschule, Berlin, 1944. ( Sci. Rep Nat. Tsinghua Univ., Ser A. 259-349 (1949))

[2] W.Z. Qian and S.C. Liang, Complex form equation and asymptotic solution. J. of Tsinghua University, 19(1979)(1) 27-47,

[3] Z.H. Xia and W. Zhang, The general solution for thinwalled curved tubes with arbitrary loadings and various boundary conditions. Int. J. Pressures and Piping 26(1986) 129-144.

[4] W. Zhang, W.M. Ren and B.H. Sun, Toroidal Shells history, current situation and future. Fifth Conf. of Space Structures, Lanzhou, China 1990.

[5] R.J. Zhang and W. Zhang, Turning point solution for thin toroidal shell vibrations. Int. J. Solids Structures 27(1991)(10) 1311-1326.

[6] R.J. Zhang and W. Zhang, Toroidal shells under nonsymmetric loading. Int. J. Solids Structures, 31(1994) 27352750.

[7] Audoly, B. and Pomeau, Y., 2002. The elastic torus: anomalous stiffness of shells with mixed type, C. R. Mecanique 330, 425-432.

[8] B. Audoly and Y. Pomeau, Elasticity and Geometry. University of Cambridge, Cambridge, 2010

[9] B.H. Sun, Closed-form solution of axisymmetric slender elastic toroidal shells. J. of Engineering Mechanics, 136 (2010) 1281-1288.

[10] B.H. Sun, Toroidal Shells. (Nova Novinka, New York, 2012)

[11] R.A. Clark, R.A. and E. Reissner, Bending of curved tubes, Advances in Applied ;Hechanics. vol.II, Academic Press (1950)

[12] R.A. Clark, On the theory of thin elastic toroidal shells. J. Mech. Phys. Solids, 29(1950) 146-178.

[13] N.C. Dahl, Toroidal-shell expansion joints. J. of Applied Mechanics, ASME,20(1953) 497-503.

[14] V.V. Novozhilov, The Theory of Thin Shell. (Noordhoff, Groningen, 1959).

[15] S. Timoshenko and S. Woinowsky-Krieger, Theory of Plates and Shells. McGraw-Hill, New York (1959).

[16] Flügge, W., Stresses in Shells. Springer-Verlag Berlin (1973).

[17] Gol'denveizer, A.L., Theory of Elastic thin Shells. Pergamon Press, New York (1961).

[18] B.H. Sun, Centenary studies of toroidal shells and in memory of Prof. Zhang Wei. Mechanics in Engineering, 37(2013). (In Chinese)

[19] Föppl, L., Vorlesungen Über Technische Mechanik. vol- ume 5.B.G. Teubner, Leipzig, Germany (1907).

[20] G. Weihs. Über Spannungs- und Formänderungszustände in dünnen. Hohlreifen. Halle a. S. 1911.

[21] H. Wissler, Festigkeiberechung von Ringsflachen. Promotionarbeit, Zurich (1916). https://doi.org/10.3929/ethza-000099037

[22] V.V. Kuznetsov and S.V. Levyakov, Nonlinear pure bending of toroidal shells of arbitrary cross-section. Int. J. of Solids and Structures, 38(2001) 7343-7354.

[23] A. Zingoni, N. Enoma and N. Govender, Equatorial bending of an elliptic toroidal shell. Thin-Walled Structures, 96(2015) 286-294.

[24] W. Jiammeepreecha and S. Chucheepsakul, Nonlinear static analysis of an underwater elastic semi-toroidal shel1. Thin-Walled Structures, 116(2017) 12-18.

[25] N. Enoma and A. Zingoni, Analytical formulation and numerical modelling for multi-shell toroidal pressure vessels. Computers and Structures, 232(2020), Article 105811.

[26] H. Reissner, Spannungen in Kugelschalen (Kuppeln). Festschrift Heinrich Müller-Breslau (A. Kröner, Leipzig, 1912), 181-193

[27] E. Meissner,Über und Elastizitat Festigkeit dunner Schalen. Viertelschr. D. nature.Ges., Bd.60, Zurich (1915).

[28] F. Tölke, Ingenieur Archiv., 9 (1938), 282

[29] E. Reissner, On bending of curved thin-walled tubes. Proc. National Academy of Sci., (1949) 204-208.

[30] L.N. Tao, On toroidal shells. J. of Math. and Physics, 38,(1959) 130-134.

[31] C.R. Steele, Toroidal pressure vessels. J. Spacecr. Rocket., 2(1965) 937-943,

[32] B.H. Sun, Exact solution of Qian's equation of slender toroidal shells. Mechanics in Engineering, 38(2018)(5) 567-569.(in Chinese)

[33] B.H. Sun. On Symmetrical Deformation of Toroidal Shell with Circular Cross-Section. Preprints 2020, 2020030156 (doi: 10.20944/preprints202003.0156.v2).

[34] A. Ronveaux, Heun's Differential Equations. Oxford University Press (1995).

[35] https://en.wikipedia.org/wiki/Heun_function

[36] J.E. Marsden, L. Sirovich and S.S. Antman eds. Hypergeometric Functions and Their Applications. Texts in Applied Mathematics. 56. New York: Springer-Verlag (1991).

[37] J.N. Reddy, Theory and Analysis of Elastic Plates and Shells. CRC Press, 2007.

[38] Marple https://www.maplesoft.com/

[39] A.W. Leissa, Vibration of Shells.Acoustical Scociety of America, 1993. 